\title{
Displacement of the non-reduction temporomandibular disk with limitation of opening. Story of a clinical case-Follow up five years
}

\author{
Gustavo Helder Vinholi ${ }^{1}$, Túlio Marcos Kalife Coêlho ${ }^{2}$, Elizeu Insaurralde ${ }^{2}$, \\ Anísio Lima da Silva ${ }^{2}$, Gustavo Adolfo Pereira Terra ${ }^{1}$, Luís Gustavo Nunes Dias de Pinho ${ }^{1}$ \\ ${ }^{1}$ Postgraduate Program in Health and Development in the Midwest Region, Medicine School, Federal University of Mato Grosso do \\ Sul, Mato Grosso do Sul, Brazil; gvinholi@yahoo.com.br \\ ${ }^{2}$ Department of Restorative Dentistry and Prosthodontics, Dental School, Federal University of Mato Grosso do Sul, Mato Grosso do \\ Sul, Brazil
}

Received 30 November 2013; revised 5 January 2014; accepted 15 January 2014

Copyright (C) 2014 Gustavo Helder Vinholi et al. This is an open access article distributed under the Creative Commons Attribution License, which permits unrestricted use, distribution, and reproduction in any medium, provided the original work is properly cited. In accordance of the Creative Commons Attribution License all Copyrights (C) 2014 are reserved for SCIRP and the owner of the intellectual property Gustavo Helder Vinholi et al. All Copyright (C 2014 are guarded by law and by SCIRP as a guardian.

\section{ABSTRACT}

Displacement of the non-reduction disk with limitation of opening (DDwLO) can be treatment of the non-surgical form. However, it required scientific and clinic knowledge of diagnostic area of the disorders temporomandibulares. This study was performed with the patient $\mathrm{CN}, 18$ years, white, female, who went to the orofacial pain ambulatory with complaints of extreme pain on the right temporomandibular joint (TMJ) and limitation of maximum opening of $29 \mathrm{~mm}$ with shift to right, which she has been suffering for at least three years. After a criterion anamnese the diagnosis hypothesis found was a displacement of the non-reduction disk with limitation of opening. The treatment was based on infiltration in the right TMJ with anesthesic followed by mandibular manipulation. After the therapeutic conduct the patient presented clinical signals of normality with absence of trismus and mandibular movement without shift to right. The patient was followed up during five years not presenting return of the pathology.

\section{KEYWORDS}

Temporomandibular Disorders; Displacement of the Disk; Temporomandibular Joint

\section{INTRODUCTION}

The term usually denotes a disk displacement (DD) abnormal position of the articular disc on the mandibular condyle and articular eminence [1].

The temporomandibular joint disorders develop an important role in the production of signs and symptoms such as clicks, pain, crepitation, and locking jaw or mandibular trismus which has been widely discussed in the literature. But its pathogenesis remains without evidence, however the second Manfredine D [2] the morphological role (occlusal abnormalities and slope of the articular eminence) and functional factors (muscle hyperactivity side pterigoideo) seem to be much less important than reported in the past. The author suggests that studies addressing the pathogenesis of DD should start with an improvement in the understanding of the mechanism of TMJ by synovial fluid lubrication and its decrease.

According to a study of the prevalence temporomandibular disorders (TMD) the authors detected that $8 \%$ of the diagnosed cases involved only TMJ, 73\% involved only the masticatory muscles and $19 \%$ had the two disturbance forms. According to this study researchers about 8 million Brazilian have some degrees of TMD 90\% of its total are women aged between 20 and 45 years [3].

The DDwLO has been by scientific definition the original disk moved of its position between the condyle and the articular fossa for a forward, medial or lateral position, associated with limitation of opening [4-6]. According to Carlsson et al. [4], Palla [7], Zarb et al. [6], LeResch et al. [5] the pathology has presented as pathognomonic signs and symptoms history of significant limitation of mouth opening, deviations of mouth opening without correction of the jaw to the side of the affected joint, absence of articulate sound, usually the 
patient reports prior history of clicks in which affected TMJ reciprocal was ceased after mandibular locking and pain in the auricular region during the function mandibular, chewing hard food, pain during palpation exams and articular functional test.

\section{MATERIALS AND METHODS}

The patient CN, 18 years, female, white, has gone to the Orofacial pain and TMD ambulatory of University of Dentistry of the Federal University of Mato Grosso do Sul with complaints of a pain in the pre-auricular right region, trismus with maximum opening of $29 \mathrm{~mm}$ and jaw movement with shift right (Figures 1 and 2), the pain began strong and intense presence 45 days ago (referring to the last pain episode-CN reported having suffered from those pains for 3 years). The quality of the pain was described as pulsating and twinge type on the right ear with a frequency of 7 days per week during the

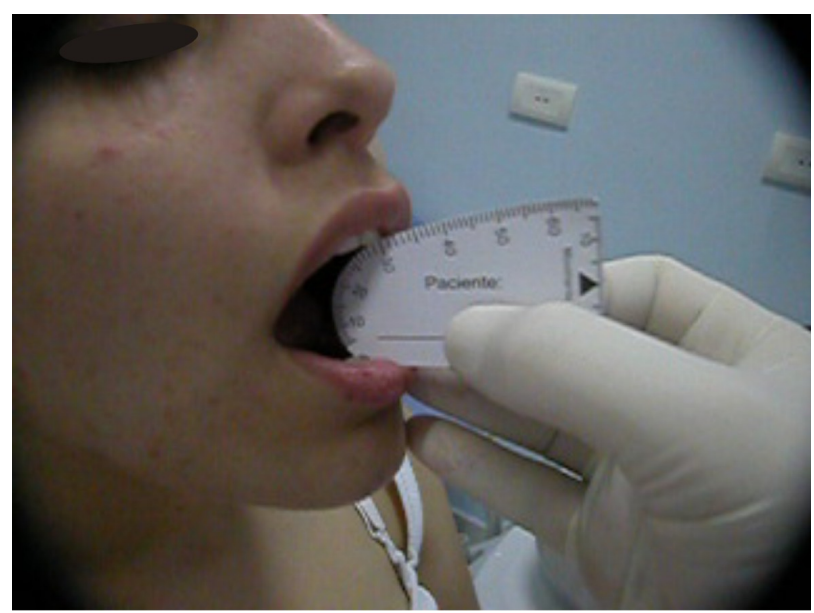

Figure 1. Limitation of mouth opening with maximum opening of $29 \mathrm{~mm}$.

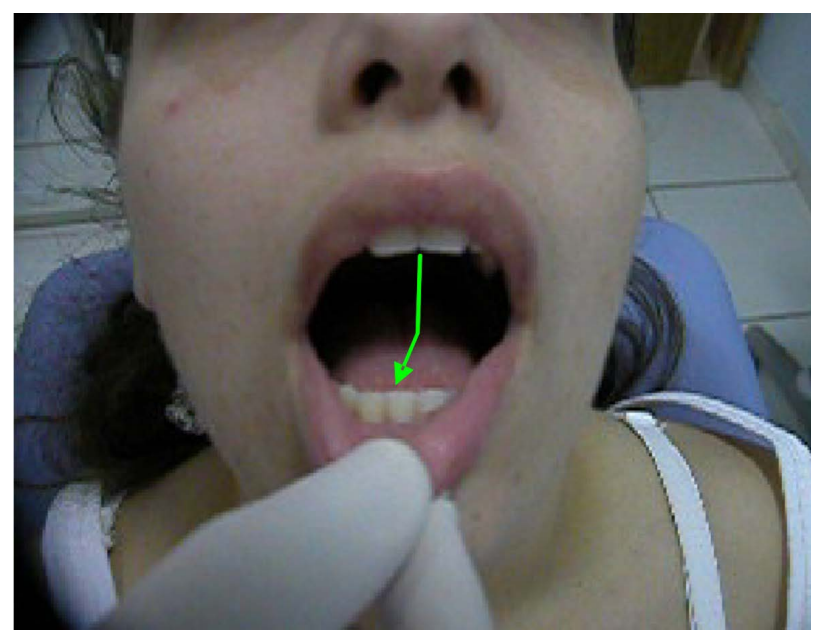

Figure 2. Limitation of mouth opening and jaw movement with deviation to the right side. whole day. It used to begin in the morning and lasted during the day until the end of the night. She informed that when she was chewing hard foods and doing any function or parafunction jaw type, the pain increased its intensity. She had as an improving factor the non accomplishment of any jaw activity as well as speaking; as worsening factor the stress, chewing hard foods and parafunction.

After criterion anamneses the probable hypothesis diagnosis for the case was DDwLO [5]. The suggested treatment consisted of intra-joint infiltration in the right TMJ followed by jaw manipulation to recapture of the joint disk which was impeding the complete translation movement of TMJ previously affected [2]. It was performed an intra-joint infiltration with Citanest ${ }^{\circledR}$ anesthetic in the quantity of $1.8 \mathrm{Ml}$ of prilocain with felipressin on $0.03 \mathrm{UI} / \mathrm{ml}$ through carpule syringe (Figure 3 ). The applied technique consisted of a modification of the arthrocentesis technique proposed by Nitzan, et al. [8] as a result it was executed the infiltration of the liquid only without the presence of the aspiration clyster-pipe. The tragus line was delimited-edge of the eye and the first demarcation $10 \mathrm{~mm}$ distant from the tragus and $0.5 \mathrm{~mm}$ below the drawn line, and after that the patient was kept with his mouth opened during the whole procedure so that the liquid could be introduced inside of the joint cavity (superior compartment) that stayed empty, the condyle was then placed forward.

After the procedure, it was done the jaw manipulation which aimed the recapture of the joint disk which was in previous position and without any reduction. A passive manipulation was executed with movements in the anticlockwise sense. In this case it was obtained success with the recapture of the disk with presence of a strong click during the process (Figure 4).

\section{RESULTS}

After the jaw manipulation a new evaluation was carried out presenting as a result the re-establishment of the jaw dynamics with opening and closing without shift, click, limitation of the opening or pain (Figures 5 and 6). The mandibular range of motion aid was of $51 \mathrm{~mm}$ without pain in the right TMJ. As a complement of the therapy it was made a front-plateau in the same session maintaining the jaw totally onward for 02 days of continuous use with the purpose of returning the disk to its original anatomy type "butterfly tie”. It was administred an non-hormonal anti-inflammatory of selective inhibition type-Arcóxia ${ }^{\circledR} 90 \mathrm{mg}$, once a day for one week and criotherapy in the right TMJ for 10 minutes, three times a day for one week.

The patient's follow-up was performed in spaces of 06 months. After five years of treatment the patient was revalued again and her mandibular opening without aid 


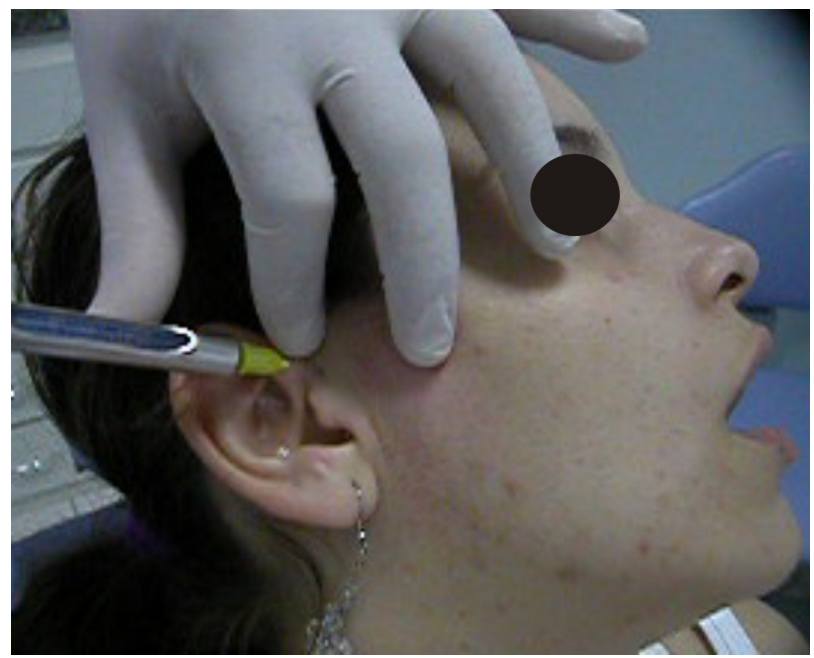

Figure 3. Infiltration of the anesthetic solution.

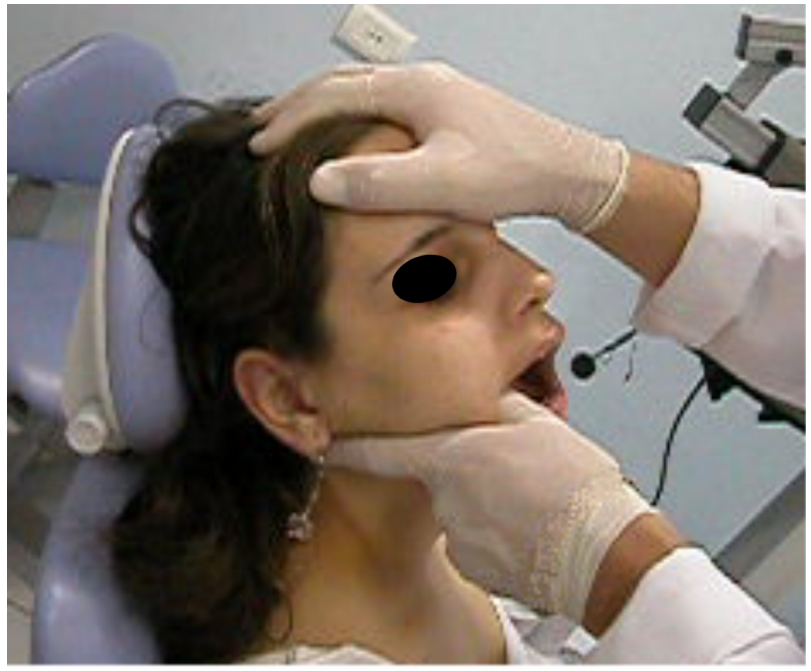

Figure 4. Mandibular manipulation.

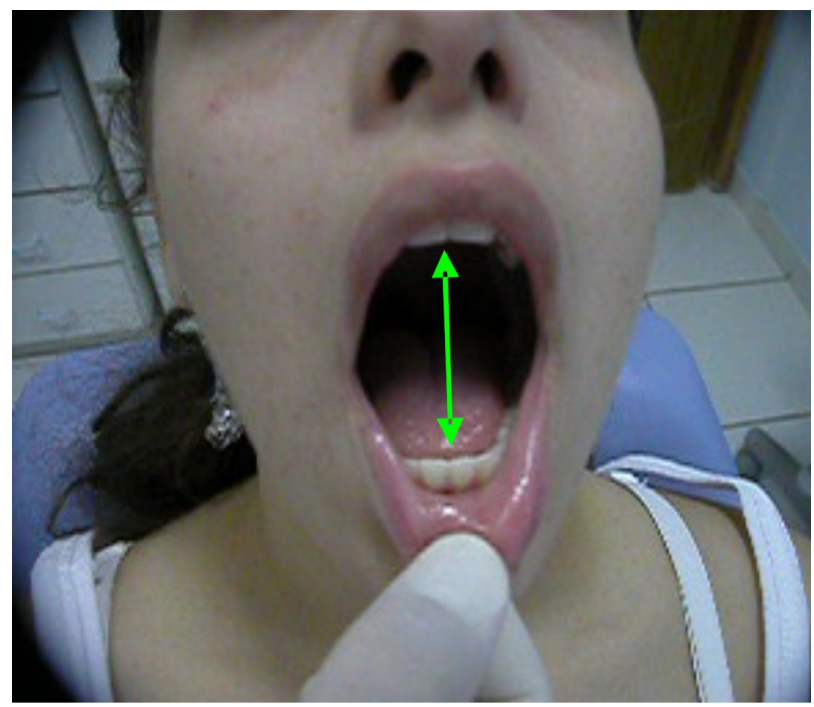

Figure 5. Re-establishment of the normal jaw dynamics.

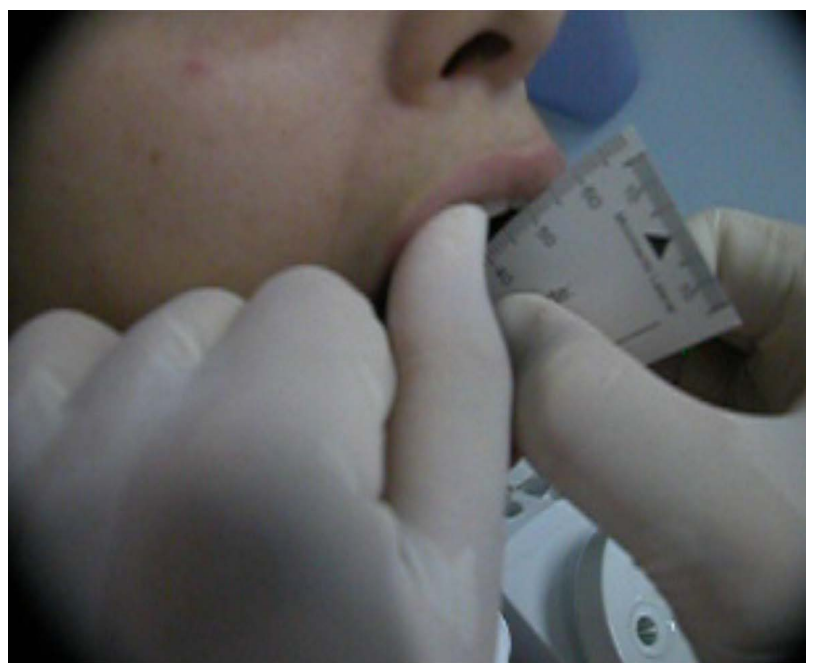

Figure 6. Mandibular motion with maximum opening of 51 $\mathrm{mm}$.

became to $52 \mathrm{~mm}$ without the click presence, shift, trismus or pain in TMJ presenting as a result the success of the chosen therapy and the non recurrence of the pathology (Figures 7 and 8).

\section{DISCUSSION}

Becomes relevant to this case because this therapy represents a conservative and reversible mode of TMJ treatment with high possibility of success in the control of this pathology with minimal collateral and undesirable effects.

This treatment protocol for internal derangement of TMJ is a new modality of non surgical. In the past, many cases of DD and jaw trismus which have been treated in a conservative way in the clinic such as plates, thermotherapy, physiotherapy, criotherapy and drugs among others, and that could not have reached the wanted success, were directed to the surgical center and submitted to more aggressive therapies through anchorage of the

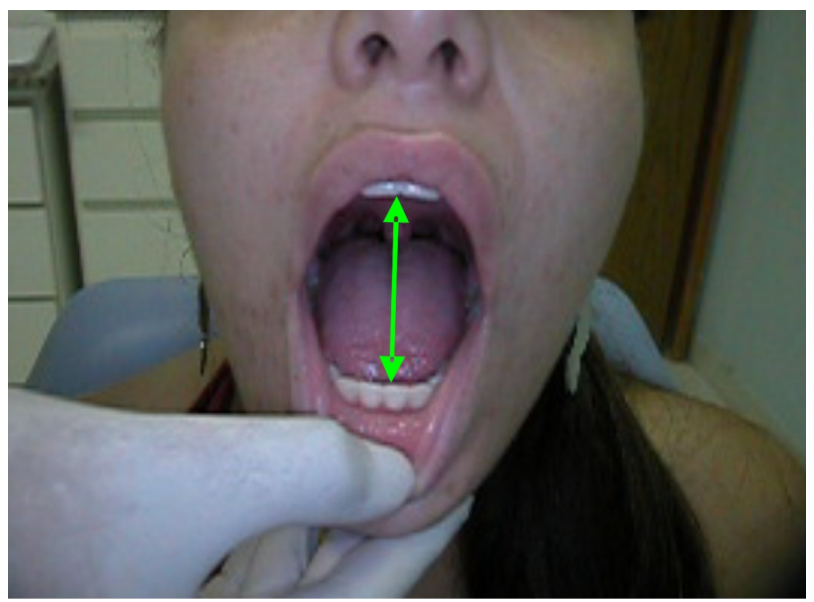

Figure 7. Mandibular motion five years after treatment. 


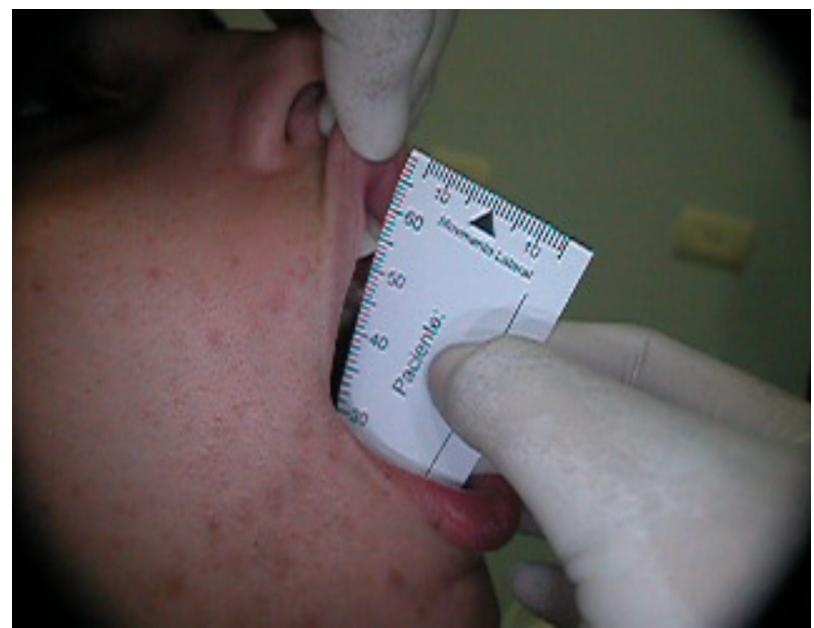

Figure 8. Maximum opening of mouth of $54 \mathrm{~mm}$ five years after treatment.

joint disk and/or arthroplastics of TMJ [9]. This therapeutic emerges as an intermediate alternative between the conservative treatment and the surgical one. The advantages of the therapy are conveyed in the simplicity of the technique applied, and also, in the fact of being completely reversible, of lower cost and executable in the dental clinic. The wash of the superior joint space reduces the pain motivated by the removal of the present inflammatory mediators in the joint [10]. There is an increasing on the jaw mobility for the break of the intra-joint adhesions [11] and for the improvement of the mobility of the disk that reduces the mechanical obstruction caused by the previous positioning of the disk [7]. The jaw manipulation takes aim at an attempt of the recapture of the disk or at least to provoke the break of the adhesions of the disk that allows the return of the lubrication by the sinovial liquid and the reabsorption of the persistent inflammatory liquid [1-7]. The previous stability plate (front-plateau) blocks the jaw in an advanced position allowing the capsular ligament to came to an stabilization and as consequence provide the remodelation of the bilaminar area and returning to its anatomical format disk of "butterfly tie" by the prolongation of the previous recess and compression of its central portion $[7,12,13]$. In this specific case it was obtained success in the improvement of the sintomathology associated to the internal derangement and an increase of the mandibular range motion.

\section{CONCLUSION}

In this case presented and in accordance with the methodology of the therapeutic protocol used, effective improvement was observed in the clinical picture of this patient with DD without reduction with limitation of the opening, suggesting that in cases like this, the treatment protocol can be used for the improvement of the clinical signs and symptoms.

\section{REFERENCES}

[1] Conti, P.C.R., Miranda, J.E. and Ornelas, F. (2000) Joint noises and signs of temporomandibular dysfunction: A comparative study by means of manual palpation and computerized vibratografia of TMJ. Pesqui Odontol Bras, 14, 367-371.

[2] Manfredini, D. (2009) Etiopathogenesis of disk displacement of the temporomandibular joint: A review of the mechanisms. Indian Journal of Dental Research, 20, 212.

[3] Bove, S.R., Guimarães, A.S. and Smith, R.L. (2005) Characterization of patients in a temporomandibular dysfunction and orofacial pain outpatient clinic. Revista LatinoAmericana de Enfermagem, 13, 686-691.

[4] Carlsson, G.E., Magnusson, T. and Guimarães, A.S. (2006) Management of the temporomandibular disorder in the General Dental Practice. 1st Edition, Quintessence, São Paulo, 215 p.

[5] LeResche, L., Fricton, J., Mohl, N., Sommers, E. and Truelove, E. (1992) Research Diagnostic Criteria. Journal of Craniomandibular Disorders: Facial \& Oral Pain, 6, 327-339.

[6] Zarb, G.A., Carlsson, G.E. and Sessle, J.B. (2000) Temporomandibular joint and Masticatory Muscle Disorders. Santos, São Paulo, 624.

[7] Palla, S. (2004) Myoarthropathy of the masticatory system and orofacial pain. Artes Médicas, 502 p.

[8] Nitzan, D.W., Dolwick, M.F. and Martinez, G.A. (1991) Temporomandibular joint arthrocentesis: A simplified treatment for severe, limited mouth opening. Journal of Oral and Maxillofacial Surgery, 49, 1163-1167. http://dx.doi.org/10.1016/0278-2391(91)90409-F

[9] Moses, J.J., Sartoris, D., Glass, R., Tanaka, T. and Poker, I. (1989) The effect of arthroscopy surgical lysis and lavage of the superior joint space on temporomandibular joint disk position and mobility. Journal of Oral and Maxillofacial Surgery, 447, 674.

http://dx.doi.org/10.1016/S0278-2391(89)80004-7

[10] Cavalcanti do Egito Vasconcelos, B., Bessa-Nogueira, R.V. and Rocha, N.S. (2006) Arthrocentesis of the temporomandibular joint: Evaluation of results and review of the literature. Revista Brasileira de Otorrinolaringologia, 72, 634-638.

[11] Spallaccia, F., Rivaroli, A. and Cascone, P. (2000) Temporomandibular joint arthrocentesis: Long-term results. Bull Group Int Rech Sci Stomatol Odontol; Belgium, 42, 31-37.

[12] Shoji, Y.N. (1995) Nonsurgical treatment of anterior disk displacement without reduction for the temporomandibular joint: A case report on the relationship between condylar rotation and translation. The Journal of Craniomandibular Practice, 13, 270-273.

[13] Farrar, W.B. and McCarty, W.L. (1982) A clinical outline of temporomandibular joint diagnosis and treatment. Normandie Publications, Montgomery. 